\title{
Antimicrobial prescribing and determinants of antimicrobial resistance: A qualitative study among physicians in Pakistan
}

\author{
Zikria Saleem ${ }^{1,2}$, Mohamed Azmi Hassali ${ }^{1}$, Brian Godman ${ }^{1,4,5,6}$, Furqan Khurshid Hashmi ${ }^{3}$, Fahad Saleem ${ }^{7}$ \\ ${ }^{1}$ School of Pharmaceutical Sciences, Universiti Sains Malaysia, Penang, Malaysia \\ ${ }^{2}$ Rashid Latif College of Pharmacy, Lahore, Pakistan \\ ${ }^{3}$ University College of Pharmacy, University of the Punjab, Lahore, Pakistan \\ ${ }^{4}$ Department of Clinical Pharmacology, Karolinska Institute, Stockholm, Sweden \\ ${ }^{5}$ Strathclyde Institute of Pharmacy and Biomedical Sciences, Strathclyde University, Glasgow, UK \\ ${ }^{6}$ Health Economics Centre, University of Liverpool Management School, Liverpool, UK \\ ${ }^{7}$ Faculty of Pharmacy and Health Sciences, University of Balochistan, Quetta, Pakistan \\ Corresponding Author \\ Zikria Saleem - School of Pharmaceutical Sciences, Universiti Sains Malaysia, Malaysia \\ Phone Number: +92322-9801981 Email ID: xikria@gmail.com
}

(Accepted for publication International Journal of Clinical Pharmacy. Please keep Confidential)

Abstract

Background: Understanding physicians' perception about antimicrobial use and resistance is essential to ensure that the objectives of the Pakistan national action plan on antimicrobial resistance are met. Little is currently known about physicians' perceptions in Pakistan. Objective: Assess physicians' perception surrounding antibiotic use and resistance, factors influencing antibiotic prescribing and potential interventions to improve future antibiotic prescribing. Settings: The study was conducted in Lahore, the capital of the province of Punjab, which is the second largest and most populous city of Pakistan.Method: Qualitative study was conducted with a semi-structured interview guide involving in-depth face-to-face interviews with purposively selected physicians. Audio-recorded interviews were transcribed verbatim and transcripts analyzed by thematic content analysis. Main Outcome Measures: Themes surrounding the perspectives of physicians on issues of antimicrobial use and resistance. Results: Five major themes emerged: (1) knowledge and perception of physicians about antimicrobials, (2) antimicrobial prescribing behaviors of physicians, (3) factors influencing prescribing, (4) determinants of antimicrobial resistance, (5) and potential interventions to reduce antimicrobial resistance. The main challenges and issues associated with antibiotic prescribing were the improvement of knowledge, implementation of hygienic measures, access to and clarity of treatment recommendations and minimizing external factors influencing prescribing including pharmaceutical company activities. Suggestions for the future included stricter regulations for prescribing, improved diagnosis availability of local guidelines and monitoring of prescribing and resistance patterns. Conclusion: Identification of concerns regarding inappropriate antimicrobial prescribing will enable 
specific initiatives and approaches to improve future antimicrobial use and reduce antimicrobial resistance in Pakistan.

Key Words: Antimicrobial resistance; Physicians; Prescribing; Qualitative study; Pakistan

\section{Impact of findings on practice}

- Physicians in Pakistan seem to have insufficient knowledge about the appropriate prescribing of antibiotics.

- To prevent inappropriate prescribing of antibiotics, prescribing guidelines in Pakistan must be strengthened and clarified.

- Improved and continual education would also reduce reliance on pharmaceutical companies for educational activities, and this will be a suggested activity building on exemplars in other countries

- These will be suggested activities to the authorities in Pakistan in order to attain the goals of the recently launched its national action plan.

\section{Introduction}

Antimicrobial resistance (AMR) is a growing problem worldwide increasing morbidity, mortality, and costs [1]. This problem is spreading predominantly in low and middle-income counties (LMICs), resulting in suggested initiatives globally, regionally, and nationally to address this [2-4]. The main factors increasing AMR rates in LMICs include the excessive use of antimicrobials as well as insufficient infection control due to poor hygiene [5]. Antibiotics are typically prescribed or dispensed inappropriately and/ or patients are given inappropriate dose regimens, enhanced by patient pressure [6,7]. Additionally, patients do not always comply with the treatment regimens, which can subsequently increase their health care costs. Moreover, physicians and pharmacists do not always have adequate knowledge regarding antibiotics [7, 8].

Reduction in inappropriate antibiotic use can be achieved by changing the prescribing behavior of physicians [9]. In this respect, antimicrobial stewardship programs (ASP) can play an important role in the optimal use of antimicrobial therapy in hospitals [10]. ASPs can reduce inappropriate antibiotic use and resistance, decrease hospital stay, and reduce health care costs [11]. Studies have shown that physicians and other key healthcare professionals are supportive of ASP because they improve the care of patients [12]. However, perceptions of physicians about ASPs as well as antibiotic use and resistance do vary across countries and sectors [7, 9, 12-17].

Studies have shown that although prescribers agree antibiotics are often used excessively, they do not always agree that antibiotics are overused in their own institutions $[13,18]$. Likewise, some physicians also believe that AMR is a national issue rather than a local one $[9,14]$. Nonetheless, the varying degree of knowledge of antibiotics among physicians offers a challenge in finding appropriate policies although emphasizing educational activities to improve 
their knowledge regarding appropriate use of antibiotics to help improve future use [7, 9, 12-18]. Countries are auditing physician adherence to antibiotic guidelines as a better way of assessing the quality of prescribing given varying rates in practice [19]. The quality of medicines in developing countries such as Pakistan, including antimicrobials, can be an issue potentially affecting AMR rates [20].

Consequently, baseline information will be crucial as a starting point for improving the effectiveness of any ASP in Pakistan, including the training of physicians on the rational use of antibiotics. As a result, the perception of physicians about the driving forces behind antibiotic prescribing and resistance is an essential first step, and such information can be acquired through qualitative research. This is important in Pakistan with only limited knowledge regarding physician attitudes towards antibiotics and AMR, and knowing that the introduction of ASPs in LMICs such as Pakistan remains a challenge [4].

\section{Aim of the Study:}

An in-depth qualitative study was conducted among Pakistani physicians to assess their perception about antibiotic use and resistance, factors influencing their prescribing of antibiotics and potential interventions to improve their future antibiotic prescribing. Such an approach provides valuable information on why physicians act as they do, and focuses on extracting underlying reasons and attitudes for their actions to guide future activities.

\section{Ethics Approval}

The study was approved by the Ethics Committee for Clinical Research of the University College of Pharmacy, University of the Punjab, Lahore, Pakistan (HEC/1000/PUCP/1925D).

\section{Method}

\section{Study design}

A qualitative study was undertaken since this design is considered to be the most appropriate method to explore a wide range of in-depth viewpoints and extract key information and ideas from healthcare professionals. Overall, qualitative research intends to synthesize views, experiences, behaviors, and opinions of an eclectic mix of respondents $[15,17]$. The COREQ (consolidated criteria for reporting qualitative research) checklist for reporting qualitative studies guided the reporting of the methods and results [21].

\section{Instrument development}

A semi-structured interview guide (A1) was developed based on an in-depth review of published papers [7, 9, 12-

18]. Before conducting any interviews, the interview guide was assessed for its validity and reliability to enhance the robustness of the findings. By applying argumentative and cumulative techniques, experts at the Universiti Sains Malaysia and the University of the Punjab with extensive experience in such interviews reviewed and validated the initial guide. All of the questions were subsequently reviewed and approved by two independent physicians working in hospitals in Pakistan to enhance the applicability of the interview guide. A pilot study was also conducted beforehand, and the interview guide improved accordingly. The reliability of the study findings was assured by recording face-to-face interviews with the physicians following their approval. 


\section{Respondents sampling and inclusion criteria}

The study was conducted in Lahore, the capital of the province of Punjab, which is the second largest and most populous city of Pakistan. Registered physicians with the Pakistan Medical and Dental Association (PMDC) were recruited using a purposive sampling technique. Only those physicians who were working in licensed health care settings and have a license issued by the Director General Health Services, Department of Health, Government of the Punjab, Lahore, Pakistan, were included, with a particular emphasis on those physicians working in tertiary hospitals who typically have a private practice as they are likely to have the broadest knowledge. The research team started with Lahore since if there were concerns with knowledge regarding antimicrobials in this capital city, such concerns are likely to be seen throughout Pakistan. However, there could be differences in responses from physicians working in cities compared with rural areas based on experiences with other healthcare professionals.

\section{In-depth face-to-face interviews}

Before the interview, the physicians who participated in this study were verbally informed about the objective of the study. The interviews were conducted one on one in a place chosen by the interviewee (office, residence, clinic, hospital). The identity of the respondents was kept confidential to ensure complete privacy. The anonymity of their opinions was confirmed by using codes. Interviews were conducted until the occurrence of saturation, a point at which no new data emerges. All interviews were conducted in English. Each interview lasted between 25 to 45 minutes. Probing questions were used, and interviewees were given liberty to describe additional views and comments. All the interviews were audio-recorded and the principal researcher (ZS) took additional field notes. Saturation was attained by the twelfth interview; however, three more interviews were conducted to ensure saturation. The fifteen interviews took place between March 2018 and June 2018. All the interviewees also gave the written consent to proceed. They were also allowed to voluntarily withdraw from the study at any time.

\section{Analysis}

Recorded interviews were transcribed verbatim, and the transcripts were written up in English. In the process of data extraction, the verbatim comments were minimally changed for grammatical errors. In the first step of data analysis, a thematic content analysis (TCA) was undertaken by three researchers (ZS, MAH, FKH) in which relevant answers from each of the transcripts relating to the general research questions were deductively highlighted. In the second step, one respondent's answers were compared and contrasted with those from other respondents to ascertain any differences. The identified patterns were subsequently reorganized, and emerging themes were identified from the interview transcripts. The analysis provided an opportunity to establish initial themes and subsequently look for divergent or negative responses, exploring our interpretation and the complexity of the data. The ideas were discussed within the research team during the course of the interviews in order to explore pertinent issues further. The research team constantly sought to keep the richness of the physicians' experiences, noting atypical cases, contradictions, and conflicts within the data. Upon categorizing a theme, the research team analyzed all the transcribed interviews for interrelated comments. This practice helped ensure cases initially observed as unrelated 
could be clustered together as their interconnectedness became apparent. In this way, the research team could be confident of the findings.

\section{Results}

The demographics of the fifteen physicians who were interviewed are given in Table 1. Most of the participants were male (73.3\%) and aged between 30-45 years (80.0\%). Following the analysis, five major themes emerged. These were (1) Knowledge and perception of physicians about antimicrobials, (2) Antimicrobial prescribing behaviors of physicians, (3) Factor influencing antimicrobial prescribing, (4) Determinants of AMR, (5) and potential interventions to control AMR.

Table 1: Demographics of respondent physicians

\begin{tabular}{llllll}
\hline Gender & $\begin{array}{l}\text { Age } \\
\text { (Years) }\end{array}$ & $\begin{array}{l}\text { Experience } \\
\text { (Years) }\end{array}$ & $\begin{array}{l}\text { Level Of } \\
\text { Qualification }\end{array}$ & Designation & $\begin{array}{l}\text { Area Of } \\
\text { Practice }\end{array}$ \\
\hline Male & 40 & 12 & FCPS & $\begin{array}{l}\text { Assistant } \\
\text { Professor }\end{array}$ & Surgery \\
Male & 32 & 6 & MBBS & $\begin{array}{l}\text { Medical Officer } \\
\text { Senior Registrar }\end{array}$ & Medicine \\
Male & 40 & 14 & FCPS & Assicine \\
Male & 40 & 13 & FCPS & Professor & Surgery \\
Female & 55 & 30 & FCPS & Professor & Medicine \\
Female & 35 & 10 & MBBS & Senior Registrar & Surgery \\
Male & 30 & 5 & MBBS & Medical Officer & Medicine \\
Female & 32 & 7 & MBBS & Senior Registrar & Medicine \\
Male & 35 & 10 & FCPS & Senior Registrar & Surgery \\
Male & 36 & 11 & FCPS & Assistant & Medicine \\
& & & Professor & Medicine \\
Male & 60 & 35 & FCPS & Professor & Surgery \\
Male & 40 & 15 & FCPS & Assistant & Professor \\
& & & Senior Registrar & Medicine \\
Male & 30 & 5 & MBBS & Senior Registrar & Surgery \\
Female & 43 & 16 & MBBS & Assistant & Medicine \\
Male & 57 & 27 & FCPS & Professor & \\
& & & & & \\
\hline
\end{tabular}

MBBS: Bachelor of Medicine and Bachelor of Surgery, FCPS: Fellow of College of Physicians and Surgeons 
Theme I: Basic knowledge and perception of physicians about antimicrobials

\section{Use of antimicrobials}

Most of the physicians said that antimicrobials are significantly overused nationally. They also confessed that there are more prescriptions of antibiotics in the hospital where they are working than necessary.

"Yes, In my hospital, I won't say they are overused, but they are used. In Pakistan, they are very much used."

[Male, 5 years experience, MBBS, Senior Registrar]

"I have been to Europe and America; there are very few physicians who are writing antibiotics and with proper culture sensitivity and with proper protocol. But in Pakistan, there is no check and balance."

[Male, 35 years experience, FCPS, Professor]

"Yes... but I used to prescribe antibiotics only when it is required."

[Male, 11 years experience, FCPS, Assistant professor]

\section{Knowledge about guidelines of antimicrobials use}

The majority of physicians did not know about any specific guidelines for antimicrobial use in their healthcare facility. Most physicians stated that they try to keep themselves updated by searching recent guidelines over the internet or by consulting some authentic books.

I mostly consult different articles, journals, book, and websites like Medscape."

[Male, 6 years experience, MBBS, Medical Officer]

"I keep myself updated through medical representatives actually. They come and educate us about different brands."

[Male, 10 years experience, FCPS, Senior Registrar]

"I usually go by the books and follow the guidelines about the appropriate selection of antibiotics."

[Male, 5 years experience, MBBS, Medical Officer]

\section{Knowledge about antimicrobial stewardship program (ASP)}

All of the physicians taking part in the interviews did not have any idea about ASPs.

I don't have an in-depth idea about it, but when I was abroad in the U.S. I did listen to a lecture about this"

[Male, 5 years experience, MBBS, Medical Officer]

\section{Perception about AMR}

All the interviewed physicians agreed that AMR was a major problem around the world as well as in Pakistan.

However, most of the interviewed physicians did not know about local resistance patterns.

"Yes, it's a problem not only in our country but in the whole world. Today I was reading an article that a multiple drug resistant typhoid is emerging in our country. So it's a grave issue."

[Male, 5 years experience, MBBS, Medical Officer]

"The resistance is not a common problem in my practice yet. But it might be in future."

[Male, 10 years experience, FCPS, Senior Registrar]

"I don't know about the pattern, but we know that there are certain patients who are resistant towards particular drug."

[Female, 10 years experience, MBBS, Senior Registrar] 
Theme II- Antimicrobial prescribing practices/behavior of physicians

Instructions on appropriate antimicrobial use

The physicians stated that they counsel the patients verbally about antibiotic use; however, only a few also gave written instructions on the proper use of medicine.

"I tell them about when to take, how to take, how much to take the medication, that you don't have to stop taking the medication when you feel better, but you have to complete the course for 5-7 days."

[Female, 10 years experience, MBBS, Senior Registrar]

"Yes. I tell the patient, esp. in case of children that the antibiotic is to be given in a proper calculated dose."

[Male, 5 years experience, MBBS, Senior Registrar]

\section{Prescribing frequency}

Most of the physicians said that they prescribe antibiotics very often depending upon the condition of the patient.

"Any patient who has any signs of inflammation requires antibiotics"

[Male, 6 years experience, MBBS, Medical Officer]

"Whenever I feel there's a risk or probability of bacterial infection, I prescribe antibiotics"

[Male, 15 years experience, FCPS, Assistant professor]

\section{Management of viral infections}

Only a few physicians said that it's very important to be aware of the fact that antibiotics are not useful for viral infections such as a common cold or flu. The physicians mostly believed that it is not necessary to determine whether the infection is bacterial or viral.

"I personally try to sort out that the infection is whether bacterial or viral."

[Male, 5 years experience, MBBS, Medical Officer]

"When a patient has a fever, we won't wait for the patient to settle down. Sometimes it's a viral infection, but we still go for antibacterial."

[Female, 10 years experience, MBBS, Senior Registrar]

"Well... In a viral infection, immunity is weak; the patient is more prone towards infections. So there I will write antibiotics."

[Male, 5 years experience, MBBS, Senior Registrar]

\section{Recommending Culture Reports}

Physicians stated that they typically prescribe antibiotics immediately without any culture report. However, in some cases such as when the patient is not showing any improvement in his/ her status or the majority of the hospitalized patients in the wards, the physicians stated they typically request culture sensitivity test reports in these situations.

"We are among the poor countries, and our socioeconomic status is not good. SO I would not let go the patient without any treatment because I know he cannot afford that particular test."

[Female, 30 years experience, FCPS, Professor]

"If it is the $1^{\text {st }}$ visit of the patient, mostly we prescribe the antibiotic. But if the patient comes again with no response, in that case, we go for culture sensitivity test to know which organism is sensitive, and then we prescribe accordingly." 
[Female, 7 years experience, MBBS, Senior Registrar]

"If the infection is in the form of discharging sinus, discharging pus, then we definitely take cultures."

[Male, 6 years experience, MBBS, Medical Officer]

"Well... Once in a blue moon, once in a month maximum."

[Male, 15 years experience, FCPS, Assistant professor]

"I am a consultant, and I prescribe antibiotics preferably according to culture sensitivity guidance and with its bacterial count."

[Male, 35 years experience, FCPS, Professor]

\section{Theme III- Factors Influencing Antimicrobial Prescribing Practices}

\section{Pressure from Patients}

A limited number of physicians said that most patients expected a medicine from the physician, and this put pressure on them to prescribe an antibiotic just to satisfy the patient. Physicians also try to explain to their patients about their condition and the fact that this condition will resolve even without the prescription for antibiotics. However, for patients who are overcautious, the interviewed physicians stated that they typically refer the patients to someone else instead of writing an antibiotic.

"The patients are in very haste to get diagnosed and treated. Even if I'll not prescribe them for quick relief, they will change the doctor"

[Male, 15 years experience, FCPS, Assistant professor]

"Yes, I guess most of the patients are not satisfied with the doctors if we don't prescribe them antibiotics. So pressure from patients is one of the factors which influence this"

[Male, 5 years experience, MBBS, Medical Officer]

"If a patient forces, then I refuse him politely or ask him to talk to some other doctor."

[Male, 15 years experience, FCPS, Assistant professor]

"Actually I am the doctor, he doesn't have the knowledge of what he wants, but I have the knowledge to see what can affect his health. I will use my knowledge instead of getting pushed by his authority."

[Female, 30 years experience, FCPS, Professor]

\section{Advice from immediate supervisor}

Most on the interviewed physicians stated that they discuss with their seniors in order to select the right choice of antibiotic; however, there is no compulsion from supervisors to write an antibiotic.

"Our supervisors are excellent in teaching us the theoretical portions and practical as well. They have no issue with the prescriptions."

[Male, 10 years experience, FCPS, Senior Registrar]

"As a consultant, my practice is independent."

[Male, 35 years experience, FCPS, Professor]

"Sometimes when there is any difficulty we seek others advice."

[Male, 13 years experience, FCPS, Assistant professor] 


\section{Influence of medical representatives}

The physicians said that medical representatives did influence prescribing; however, this is more common in private practice. The physicians also highlighted that the other important factor is the incentives for medical practitioners.

"Medical representatives have great influence because most of the physicians in the wards are not much up to date in their knowledge. Medical representatives show us their studies and trials on a specific antibiotic. It has a psychological effect on us, and we start prescribing that."

[Male, 15 years experience, FCPS, Assistant professor]

"They do visit us in a public or private sector hospital, but I do not prescribe antibiotics as per their wishes."

[Male, 13 years experience, FCPS, Assistant professor]

\section{Unhygienic and unsterilized conditions}

All the physician interviewees said that generally the cleanliness or hygiene of the wards and operation theaters is sub-optimal. Consequently, to prevent post-operative and nosocomial infections, prophylactic antibiotics are typically given. The interviewed physicians also said that doctors, nurses, and paramedics should be very strictly trained about antiseptic measures.

"We think that our operative environment is not clean up to the par, and the hygiene of the patient is not good. We think that it is important to give antibiotics to our patient in such cases."

[Male, 6 years experience, MBBS, Medical Officer]

"Because whenever a patient is about to undergo surgery, he is at high of infections particularly in our setup where septic measures are not up to date in our operation theaters and wards"

[Male, 5 years experience, MBBS, Medical Officer]

\section{Cost and Quality of medicine}

There was a mixed response to the cost and quality of medicines. The physicians said they preferred to prescribe the brand leader (typically the best-selling antibiotic with that originator name). However, if the patient can't afford the brand leader, then they would prescribe alternatives. Most physicians believed that expensive drugs were more effective.

"I always go for whatever is good and in a benefit for the patient. I don't go for the cost. Even if a medication is of low cost but is effective, I would prescribe it."

[Female, 30 years experience, FCPS, Professor]

"We are dealing with many poor people and cost is a very important issue to ensure compliance from patients"

[Male, 5 years experience, MBBS, Medical Officer]

"Certainly there are local companies which are not producing the drugs up to the mark."

[Male, 35 years experience, FCPS, Professor]

Theme IV-Determinants of AMR

\section{Unnecessary prescribing of antimicrobials}

According to most of the physicians interviewed, one of the main reasons for AMR may be unnecessary antimicrobial prescribing. It is becoming a significant problem to prescribe broad-spectrum antimicrobials unnecessarily. 
"Excessive injudicious use of antibiotics is the main reason of resistance."

[Male, 6 years experience, MBBS, Medical Officer]

"Prescribing an antibiotic again $n$ again in a patient who doesn't really need it is main reason.... After repeated exposures, it can lead to resistance."

[Female, 7 years experience, MBBS, Senior Registrar]

\section{Patients' behavior}

Socioeconomic factors are important in determining irrational practices. Most of the time, patients take fewer antibiotics than the full prescribed course because they cannot afford the full course.

"People don't know about the duration of antibiotics. They stop taking the medication if they get cured after 2 days. They are not completing its time duration, which is required according to the prescription. Ultimately that medicine becomes non-effective for them."

[Female, 10 years experience, MBBS, Senior Registrar]

According to most physicians, one of the main reasons for irrational antimicrobial use and subsequent AMR is patients' concerns with the health care system and the easy availability of antibiotics

General practitioners and quacks are the main sources of spreading drug resistance in our society. They have little knowledge of the drugs....go to the clinic and start prescribing antibiotics. The time a patient reaches the consultant he is already having resistance"

[Male, 5 years experience, MBBS, Senior Registrar]

"There is the easy availability of the antibiotics to the general public, they go the medical store and ask them this is the problem, and get without a prescription."

[Male, 13 years experience, FCPS, Assistant professor]

Theme V-Potential interventions to control AMR

Law and legislation for antimicrobial use

The interviewed physicians agreed that we could not control AMR without the implementation of law and legislation for antibiotic use. The respondents also pointed out a few strategies to control the emergence of AMR by involving all key stakeholders of health care systems such as physicians, nurses, and pharmacists.

"I think it's high time that the policymakers and the health department should give adequate attention to this rising issue of antibiotic resistance and unnecessary prescription of other medicines as well apart from antibiotics."

[Male, 5 years experience, MBBS, Medical Officer]

"Government has made narcotics prescription bound likewise the availability of all the antibiotics should also be ensured on the base of prescription."

[Male, 11 years experience, FCPS, Assistant professor]

"Government should make it possible like that no medical representative should give any present to a doctor of more than like 5 pounds which is 1000-2000 rupees."

[Male, 13 years experience, FCPS, Assistant professor]

Improve diagnostic skills 
Most of the interviewed physicians insisted that the most important strategy to improve antibiotic use is better diagnostic skills for infectious diseases.

"We need to do the right diagnosis, and then we can look up for the treatment in the book. When the diagnosis is not right, you go for hit and trial method."

[Male, 11 years experience, FCPS, Assistant professor]

"Antibiotics should be prescribed only when the infection is confirmed through investigations and culture sensitivity report that which antimicrobial is sensitive."

[Male, 15 years experience, FCPS, Assistant professor]

\section{Continuous medical education (CME) programs}

Most of the interviewed physicians agreed that relevant conferences, workshops, and continuous education programs could play a significant role to improve future antibiotic prescribing to overcome the threat of antimicrobial resistance.

"Workshops should be conducted for the physicians in which pharmacists are involved and guidelines should be given to those who are not in regular touch with studies, there should be refreshers to tell them about the prevailing conditions of resistance and how to overcome that"

[Female, 10 years experience, MBBS, Senior Registrar]

"To teach our young doctors to have their training programs and training lectures and they should use their right and power of prescribing the empirical use of antibiotics as less as possible"

[Male, 35 years experience, FCPS, Professor]

\section{Public awareness}

The interviewed physicians stated that we could fight AMR and unnecessary use of antibiotics by spreading awareness among patients. Patients should be educated to not pressurize physicians to prescribe antibiotics unless they are necessary.

"I think what people need is awareness of any kind of illness they are having."

[Female, 30 years experience, FCPS, Professor]

“Apart from doctors, it's very important to make awareness among the patients as well that can be conducted through TV channels, radio or social media."

[Male, 5 years experience, MBBS, Medical Officer]

"Self-medication certainly should be discouraged at all levels through public awareness." [Male, 11 years experience, FCPS, Assistant professor]

\section{Discussion}

To the best of our knowledge, this is the first time such an in-depth study has been undertaken in Pakistan surrounding physicians' knowledge and attitudes regarding antibiotics and AMR. This is important given that Pakistan is a highly populated country among LMICs and the irrational prescribing and dispensing of antibiotics is a concern [4]. The study identified considerable concerns regarding the knowledge and attitude of physicians about antimicrobial use. The main challenges and issues were improved knowledge, implementation of hygienic measures, access to and clarity of treatment recommendations and minimizing external factors including pharmaceutical 
company influence. The findings will be used to guide future policies in Pakistan, which is important as Pakistan develops and refines its national action plan to tackle AMR [4].

Studies have highlighted that practitioners rarely change their clinical practices unless they are both aware of, and in agreement with, the changes that are being suggested. Physicians typically need to be subjected to multiple measures and initiatives to effect changes in their prescribing, especially given the different factors affecting antibiotic utilization and AMR $[7,22]$. Of concern in this study was that knowledge of antimicrobial guidelines, antimicrobial stewardship, and the basic epidemiology of AMR was missing among interviewees in our capital city. Health care providers need to be empowered with the knowledge to understand best practices in their field rather than depending on external information sources such as pharmaceutical representatives with the bias that can be provided in the information supplied and its subsequent influence on prescribing [23]. The WHO and others have also sought to address key issues regarding appropriate prescribing with the development of a competency framework [24]. Up-todate guidelines are also important to address knowledge gaps to improve future antibiotic use and combined with educational initiatives to improve adherence to nationally agreed prescribing guidelines [7,19]. This is essential especially in LMICs such as Pakistan with a number of physicians stating they receive most of their prescribing information from pharmaceutical companies, which is similar to other studies in Pakistan and other LMICs [23, 25]. Having said this, a number of pharmaceutical companies are now becoming involved in information about AMR, at least in LMICs. While they bring much-needed resources and expertise for training, this presents a significant conflict of interest and needs to be pro-actively addressed. Availability and active dissemination of national guidelines are also important to enhance their subsequent utilization, building on examples in other countries [19].

Our study also highlighted that the majority of physicians agreed that while other physicians overprescribe antibiotics, they did not feel that their own prescribing practice was a problem. This result is consistent with previous surveys $[9,18,26]$. AMR was also less recognized as a problem in the physicians' own practice, similar to other studies $[13,14,27]$. Excessive and irrational antibiotic use was believed to be the main reasons for AMR, whereas only a few physicians believed that restricting antibiotic prescribing could be effective in reducing AMR. This inconsistency may be due to a lack of awareness of the effectiveness of policies to improve antibiotic utilization and reduce AMR [11], and physicians considering the risk of AMR as more theoretical than concrete. Addressing such misunderstandings is key to successfully reduce AMR in the future. The instigation of educational activities among physicians in Pakistan should help reduce inappropriate antibiotic prescribing and help achieve the goals of the National Action Plan (NAP) on AMR [4]. This can include potential quality indicators as well as future monitoring of antibiotic prescribing as part of AMS programs and other initiatives.

The results of our study are also consistent with a previous study in Peru, where there were concerns that the antibiotics available in the hospital were of poor quality, i.e. substandard or spurious [28]. This is perhaps not surprising given concerns with the manufacture of generics in Pakistan [20]. Such practices need to be addressed through improved surveillance and other measures to improve the quality of medicines available in Pakistan. This is important given the extent of co-payments for medicines in Pakistan with a proportion of the physicians reported 
that the cost of therapy sometimes influenced their prescribing decisions [18, 25]. In addition, there were concerns that patients were not taking the full course of antibiotics due to cost concerns. Moreover, junior physicians tended to seek advice from their senior colleagues when prescribing, irrespective of their specialty, correlating with the findings from previous studies where senior residents were more confident about the optimal use of antimicrobials compared with junior ones $[7,13]$. This discrepancy needs to be factored into any future educational program. The physician interviewees also blamed the unsterilized conditions of wards and operating theaters as the main driving factor for excessive prophylactical antibiotic use to prevent surgical site and nosocomial infections. This is seen in other LMICs with extended use of prophylaxis in practice to help prevent surgical site infections. The weak endorsement of poor hand hygiene as a contributing factor of infections may also reveal a lack of awareness of the effectiveness of this simple, though underused, practice. Consequently, simple measures such as basic hand hygiene measures need to be urgently introduced in all hospitals in Pakistan where these practices currently do not exist, with their use regularly monitored. Such initiatives will be monitored in the future.

Most of the physicians interviewed indicated that inflammation with fever was associated with antimicrobial prescribing in their practice even though there is evidence that this use is not indicated. The link between patients' desire for quick relief and antimicrobial misuse was also identified, which again needs to be addressed especially for predominantly viral self-limiting infections. Most of the physicians in this study were aware of evidence of the limited effect of antibiotic treatment for predominantly viral infections but wanted to be seen as active and signal sympathy with the patients, were subject to time pressures, feared losing their patients to other doctors as well as medicolegal concerns, which is similar to other studies [7]. These issues also need to be addressed through educational and other activities with both physicians and patients. Similarly addressing issues concerning taking the full course of antibiotics prescribed.

A number of good suggestions were made by the physicians to address concerns with the rational use of antibiotics in Pakistan. These included stricter regulations and monitoring of all aspects of antibiotic use in Pakistan, including procurement, current prescribing and dispensing practices across the sectors, as well as legal responsibilities. Pakistan can learn from both developed and developing countries as it moves forward with its NAP to reduce AMR [4]. The participating physicians also endorsed the fact that excessive and inappropriate use of antibiotics can be minimized by using more rapid and better diagnostic techniques. In addition, comprehensive knowledge of antibiotics is essential to improve their future use and the interviewed physicians stated they would like more feedback and education on appropriate antibiotic use in the future, similar to recommendations in other studies [12, $13,26]$. Availability of local guidelines, advice from infectious control teams, educational sessions as well as audit and feedback strategies were also greatly appreciated by physicians, consistent with an Australian survey on antibiotic approval and stewardship [12]. Hospital pharmacists can play a role here with physician education as well as monitoring antibiotic utilization, similar to other countries. This includes a role with developing and implementing ASPs [29]. Educational interventions carried out at the time of prescribing, potentially through clinical decision support systems and computerized physician order entry, are also considerations for the future [30]. 
Some physicians also felt that their clinical practice would be improved if they were provided with information on local AMR patterns. To address this, there are plans for the WHO prototype curriculum on AMR, which is currently in development and aligned with the competency framework, to be introduced in Pakistan to outline in detail epidemiological learning needs particularly for prescribers of antimicrobials. This builds on the recent NAP in Pakistan. As a result, physicians in the future should have a better understanding of key issues, including ways to reduce AMR. Additionally, in accordance with the objective of the global action plan on AMR, physicians will be encouraged to raise awareness among policymakers, practitioners, patients, and the general public on AMR. Pharmacists can play a key role in this, similar to other countries. This includes guiding patients on possible alternative medicines to antibiotics for essentially self-limiting diseases. Such activities will continue to be monitored in Pakistan.

A limitation of this survey is that physicians who decided not to take part may have views considerably different from those who did, possibly leading unmeasured bias. However, the results of this study are promising and our methodology to comprehensively explore different themes should have reduced this bias. Another possible limitation of this study is the inclination of physicians to give socially desirable answers instead of revealing their true opinions. This problem was minimized by assuring physicians of complete privacy. However, future qualitative studies can give further insights on this topic. In addition, the study was conducted in a single city of a large and extremely diverse country, including city versus rural areas, and the number of physicians was relatively small. Nevertheless, based on our methodology this baseline information will be crucial with improving the effectiveness of any ASP in Pakistan, including the training of physicians on the rational use of antibiotics. Moreover, as mentioned, our findings are robust in view of the methodology involved providing direction for the future policies in Pakistan, which is important as Pakistan develops and refines its national action plan to tackle AMR.

\section{Conclusion}

According to the interviewed physicians, the main challenges associated with antibiotic prescribing were the lack of knowledge, the lack of implementation of hygienic measures, access to and clarity of treatment recommendations, as well as a need to minimize external factors on physicians. Identification of knowledge and attitudes related to inappropriate antimicrobial prescribing will allow specific interventions to be designed, with the objective of targeting areas of concern to improve future antimicrobial use in Pakistan and reduce AMR. The suggested activities will be monitored in the future to help attain the goals of the Pakistan NAP to help reduce AMR.

\section{Acknowledgment}

Authors acknowledge the support of all the interviewees who participated in the study.

\section{Conflicts of Interest}

The authors declare that they have no competing interests.

\section{Funding}

There was no external funding for this project, with the study funded by the co-authors. 


\section{References:}

1. Gandra S, Barter D, Laxminarayan R. Economic burden of antibiotic resistance: how much do we really know? Clinical Microbiology and Infection. 2014;20(10):973-9.

2. Jinks T, Lee N, Sharland M, Rex J, Gertler N, Diver M et al. A time for action: antimicrobial resistance needs global response. Bulletin of the World Health Organization. 2016;94(8):558-A.

3. Saleem Z, Hassali MA. Travellers take heed: Outbreak of extensively drug resistant (XDR) typhoid fever in Pakistan and a warning from the US CDC. Travel medicine and infectious disease. 2019;27:127.

4. Saleem Z, Hassali MA, Hashmi FK. Pakistan's national action plan for antimicrobial resistance: translating ideas into reality. The Lancet Infectious Diseases. 2018;18(10):1066-7.

5 . Okeke IN. Poverty and root causes of resistance in developing countries. Antimicrobial resistance in developing countries. Springer; 2010. p. 27-35.

6. Ceyhan M, Yildirim I, Ecevit C, Aydogan A, Ornek A, Salman N et al. Inappropriate antimicrobial use in Turkish pediatric hospitals: a multicenter point prevalence survey. International Journal of Infectious Diseases. 2010;14(1):e55-e61.

7. Md Rezal RS, Hassali MA, Alrasheedy AA, Saleem F, Md Yusof FA, Godman B. Physicians' knowledge, perceptions and behaviour towards antibiotic prescribing: a systematic review of the literature. Expert review of anti-infective therapy. 2015;13(5):665-80.

8. Pakyz AL, Moczygemba LR, VanderWielen LM, Edmond MB, Stevens MP, Kuzel AJ. Facilitators and barriers to implementing antimicrobial stewardship strategies: results from a qualitative study.

American journal of infection control. 2014;42(10):S257-S63.

9. Pulcini C, Williams F, Molinari N, Davey P, Nathwani D. Junior doctors' knowledge and perceptions of antibiotic resistance and prescribing: a survey in France and Scotland. Clinical Microbiology and Infection. 2011;17(1):80-7.

10. Brink AJ, Messina AP, Feldman C, Richards GA, Becker PJ, Goff DA et al. Antimicrobial stewardship across 47 South African hospitals: an implementation study. The Lancet Infectious Diseases. 2016;16(9):1017-25.

11. Ashiru-Oredope D, Sharland M, Charani E, McNulty C, Cooke J. Improving the quality of antibiotic prescribing in the NHS by developing a new Antimicrobial Stewardship Programme: Start Smart-Then Focus. Journal of antimicrobial chemotherapy. 2012;67(suppl_1):i51-i63.

12. Bannan A, Buono E, McLaws ML, Gottlieb T. A survey of medical staff attitudes to an antibiotic approval and stewardship programme. Internal medicine journal. 2009;39(10):662-8.

13. Srinivasan A, Song X, Richards A, Sinkowitz-Cochran R, Cardo D, Rand C. A survey of knowledge, attitudes, and beliefs of house staff physicians from various specialties concerning antimicrobial use and resistance. Archives of internal medicine. 2004;164(13):1451-6.

14. Wester CW, Durairaj L, Evans AT, Schwartz DN, Husain S, Martinez E. Antibiotic resistance: a survey of physician perceptions. Archives of internal medicine. 2002;162(19):2210-6.

15. Kaae S, Malaj A, Hoxha I. Antibiotic knowledge, attitudes and behaviours of Albanian health care professionals and patients-a qualitative interview study. Journal of pharmaceutical policy and practice. 2017;10(1):13.

16. Reynolds $L$, McKee M. Factors influencing antibiotic prescribing in China: an exploratory analysis. Health Policy. 2009;90(1):32-6. doi:10.1016/j.healthpol.2008.09.002.

17. Velasco E, Ziegelmann A, Eckmanns T, Krause G. Eliciting views on antibiotic prescribing and resistance among hospital and outpatient care physicians in Berlin, Germany: results of a qualitative study. BMJ open. 2012;2(1):e000398.

18. Abbo L, Sinkowitz-Cochran R, Smith L, Ariza-Heredia E, Gómez-Marín O, Srinivasan A et al. Faculty and resident physicians' attitudes, perceptions, and knowledge about antimicrobial use and resistance. Infection Control \& Hospital Epidemiology. 2011;32(7):714-8. 
19. Nakwatumbah S, Kibuule D, Godman B, Haakuria V, Kalemeera F, Baker A et al. Compliance to guidelines for the prescribing of antibiotics in acute infections at Namibia's national referral hospital: a pilot study and the implications. Expert review of anti-infective therapy. 2017;15(7):713-21.

20. Babar A, Khan B, Godman B, Hussain S, Mahmood S, Aqeel T. Assessment of active pharmaceutical ingredients in drug registration procedures in Pakistan: implications for the future. Generics and Biosimilars Initiative Journal. 2016;5(4):156-63.

21. Tong A, Sainsbury P, Craig J. Consolidated criteria for reporting qualitative research (COREQ): a 32item checklist for interviews and focus groups. International journal for quality in health care. 2007;19(6):349-57.

22. Cabana MD, Rand CS, Powe NR, Wu AW, Wilson MH, Abboud P-AC et al. Why don't physicians follow clinical practice guidelines?: A framework for improvement. Jama. 1999;282(15):1458-65.

23. Fadare JO, Oshikoya KA, Ogunleye OO, Desalu OO, Ferrario A, Enwere OO et al. Drug promotional activities in Nigeria: impact on the prescribing patterns and practices of medical practitioners and the implications. Hospital Practice. 2018;46(2):77-87.

24. Organization WH. WHO competency framework for health workers' education and training on antimicrobial resistance: World Health Organization2018.

25. Riaz H, Godman B, Hussain S, Malik F, Mahmood S, Shami A et al. Prescribing of bisphosphonates and antibiotics in Pakistan: challenges and opportunities for the future. Journal of Pharmaceutical Health Services Research. 2015;6(2):111-21.

26. Guerra CM, Pereira CAP, Neto ARN, Cardo DM, Correa L. Physicians' perceptions, beliefs, attitudes, and knowledge concerning antimicrobial resistance in a Brazilian teaching hospital. Infection Control \& Hospital Epidemiology. 2007;28(12):1411-4.

27. Giblin TB, Sinkowitz-Cochran RL, Harris PL, Jacobs S, Liberatore K, Palfreyman MA et al. Clinicians' perceptions of the problem of antimicrobial resistance in health care facilities. Archives of internal medicine. 2004;164(15):1662-8.

28. García C, Llamocca LP, García K, Jiménez A, Samalvides F, Gotuzzo E et al. Knowledge, attitudes and practice survey about antimicrobial resistance and prescribing among physicians in a hospital setting in Lima, Peru. BMC clinical pharmacology. 2011;11(1):18.

29. Haque A, Hussain K, Ibrahim R, Abbas $Q$, Ahmed SA, Jurair H et al. Impact of pharmacist-led antibiotic stewardship program in a PICU of low/middle-income country. BMJ Open Qual. 2018;7(1):e000180.

30. Kaushal R, Shojania KG, Bates DW. Effects of computerized physician order entry and clinical decision support systems on medication safety: a systematic review. Archives of internal medicine.

2003;163(12):1409-16. 